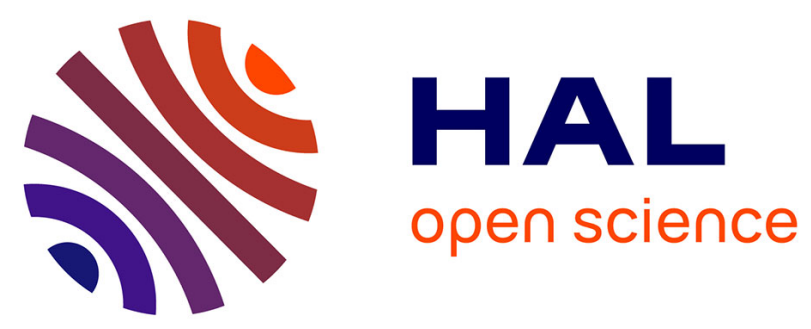

\title{
COMPORTEMENT PHYSICO-CHIMIQUE DE Si3N4 OBTENU PAR LPCVD SOUMIS A DES FLUX RADIATIFS INTENSES
}

\author{
Anqing Wang, D. Thenegal, C. Royere, C. Dupuy, B. Armas
}

\section{To cite this version:}

Anqing Wang, D. Thenegal, C. Royere, C. Dupuy, B. Armas. COMPORTEMENT PHYSICOCHIMIQUE DE Si3N4 OBTENU PAR LPCVD SOUMIS A DES FLUX RADIATIFS INTENSES. Journal de Physique Colloques, 1989, 50 (C5), pp.C5-353-C5-362. 10.1051/jphyscol:1989542 . jpa00229565

\section{HAL Id: jpa-00229565 https://hal.science/jpa-00229565}

Submitted on 1 Jan 1989

HAL is a multi-disciplinary open access archive for the deposit and dissemination of scientific research documents, whether they are published or not. The documents may come from teaching and research institutions in France or abroad, or from public or private research centers.
L'archive ouverte pluridisciplinaire HAL, est destinée au dépôt et à la diffusion de documents scientifiques de niveau recherche, publiés ou non, émanant des établissements d'enseignement et de recherche français ou étrangers, des laboratoires publics ou privés. 
JOURNAL DE PHYSIQUE

Colloque C5, supplement au $n^{\circ} 5$, Tome 50, ma1 1989

C5-353

\title{
COMPORTEMENT PHYSICO-CHIMIQUE DE $\mathrm{SI}_{3} \mathrm{~N}_{4}$ OBTENU PAR LPCV SOUMIS A DES FLUX RADIATIFS INTENSES
}

A. WANG, D. THENEGAL, C. ROYERE, C. DUPUY and B. ARMAS

Institut de science et de Génie des Matériaux et Procédés, CNRS, BP. 5, ode111o, F-66120 Font-Romeu, France

Résumé - Deux variétés de $\mathrm{Si}_{3} \mathrm{~N}_{4}, I^{\prime}$ une amorphe, I'autre cristallisée, sont élaborés sous forme de revêtements de PDSN (Pyrolytic Deposited


traitements thermiques de ces couches sur leur substrat, en régime transitoire, à I'air libre, obtenus à I'aide de creneaux de flux radiatifs intenses (limités a $450 \mathrm{~W} / \mathrm{cm}^{2}$ ) permettent de mettre en evidence des comportements physico-chimiques distincts pour les deux variétés.Le materiau amorphe est transformé en partie dès $1700^{\circ} \mathrm{C}$ en $\mathrm{Si}_{3} \mathrm{~N}_{4} \alpha$ puis dès $1800^{\circ} \mathrm{C}$ en un mélange de $\mathrm{Si}_{3} \mathrm{~N}_{4} \alpha$ et $\beta$. On observe une fissuration très dense de ce dépôt dès $1350^{\circ} \mathrm{C}$. Le matériau cristallisé $\left(\mathrm{Si}_{3} \mathrm{~N}_{4} \alpha\right)$ ne subit pas de changement de structure jusgu'a 2030: C au moins. Il se fissure à partir de $1740^{\circ} \mathrm{C}$. Les deux variétés subissent en surface, à haute température, une décomposition et une oxydation, qui affectent les morphologies des surfaces.

\begin{abstract}
The $\mathrm{Si}_{3} \mathrm{~N}_{4}$ amorphous and crystalline phases are prepared, in the shape of $200 \mathrm{~mm}$ thick PDSN coatings on graphite substrates. Heat treatments of these layers on their substrates, in air, under non steady conditions derived from high radiant heat pulses (up to $450 \mathrm{w} / \mathrm{cm}^{2}$ lead to two distinct physico-chemical behaviours of the two phases. The amorphous material starts to change partly into $\alpha \mathrm{Si}_{3} \mathrm{iJ}_{4}$ at $1700^{\circ} \mathrm{C}$ then, above $1800^{\circ} \mathrm{C}$, into a mixture of $\alpha$ and $\beta-\mathrm{Si}_{3} \mathrm{~N}_{4}$. Intense cracking of this material is observed starting at $1350^{\circ} \mathrm{C}$. The crystalline material ( $\left.\alpha \mathrm{Si}_{3} \mathrm{~N}_{4}\right)$ shows no phase transformation at least up to $2030^{\circ} \mathrm{C}$. Cracking starts at $1740^{\circ} \mathrm{C}$. At high temperature both phases turn to be surface decomposed and oxidised : these two processes modify the layers surface morphology.
\end{abstract}

\section{1-INTRODUCTION}

De nombreux travaux ont eté consacrés au comportement thermomécanique des réfractaires. La bibliographie est abondante dans ce domaine. Nous donnerons à titre d'exemple quelques références [1 à 4 ].

L'étude de la résistance au choc thermique sous rayonnement [5] a souvent fait appel à la technique de concentration du rayonnement par des fours solaires [6][7][8] : cette technique permet en effet d'imposer à la surface du matériau étudié un flux d'énergie contrôlé en niveau de flux surfacique et en durée d'exposition grâce à des dispositifs atténuateurs-obturateurs appropriés [9].

L'objectif de ce travail est de comparer les modifications physico-chimiques, induites par des variations de température résultant de créneaux de flux surfaciques, dans des matériaux réfractaires élaborés dans des conditions différentes. Les limites de flux et de température étant fixées a pxiori en deçà des conditions qui entraineraient une dégradation mécanique globale notable.

Notre choix s'est porté sur le nitrure de silicium considéré souvent comme un matériau performant pour les applications à haute température : de nombreux travaux ont été consacrés à 1 'étude de son comportement thermomécanique [10][11] et de son oxydation [12][13][14]. En revanche les modifications physico-chimiques induites par des traitements thermiques sévères, n'entrainant pas la rupture, n'ont pas été étudiées. 
De plus, suivant le mode de fabrication les propriétés du $\mathrm{Si}_{3} \mathrm{~N}_{4}$ peuvent varier considérablement [11]. Par dépôt chimique en phase gazeuse, on obtient le PDSN (Pyrolytic Deposited Silicon Nitride). C'est une technique d'élaboration très intéressante car elle permet de contrôler la microstructure du matériau et par conséquent ses propriétés thermomécaniques. Le plus fréquemment les études sur le nitrure de silicium ont porté sur le HPSN (Hot Pressed Silicon Nitride) et le RBSN (Reaction Bonded Silicon Nitride) [11].

Nous avons donc fixé, pour cette étude, notre choix sur le PDSN.

\section{2-SYNTHESE ET CARACTERISATION DE REVETEMENTS DE $\mathrm{Si}_{3} \underline{\mathrm{N}}_{4}$}

L'appareillage à paroi froide utilisé est semblable à celui qui avait conduit à l'élaboration du carbure de silicium [15] par décomposition thermique du tétraméthylsilane (T.M.S) sous pression réduite (400 Pa) et à haute température $\left(1450^{\circ} \mathrm{C}\right)$. Par la suite, nous avons utilisé le même dispositif pour étudier les conditions de préparation du nitrure de silicium [16] à partir d'un mélange de TMS et d'ammoniac $\mathrm{NH}_{3}$

Les conditions expérimentales ont été optimisées pour permettre la synthèse en couches d'épaisseur uniforme sur un substrat en graphite de $16 \mathrm{~mm}$ de diamètre et $8 \mathrm{~mm}$ d'épaisseur.

Nous avons réalisé deux séries de synthèses qui ont été conduites à des temperatures différentes dans le but d'obtenir deux types de materiaux. Ainsi à $1100^{\circ} \mathrm{C}$ est élaboré du $\mathrm{Si}_{3} \mathrm{~N}_{4}$ amorphe. Les conditions de préparation et les épaisseurs de dépôt correspondantes sont portées sur le Tableau I.

TABLEAU I

\begin{tabular}{|c|c|c|c|c|c|c|}
\hline $\begin{array}{c}\text { Echantilion } \\
\mathrm{N}^{*}\end{array}$ & $\begin{array}{c}\text { Température } \\
(\cdot \mathrm{C})\end{array}$ & $\begin{array}{c}\text { Pression } \\
(\mathrm{Pa})\end{array}$ & $\begin{array}{c}\mathrm{NH}_{3} \\
\mathrm{~N} . \mathrm{cm}^{3} / \mathrm{min}\end{array}$ & $\begin{array}{c}\text { T.M.S } \\
\mathrm{N} . \mathrm{cm}^{3} / \mathrm{min}\end{array}$ & $\begin{array}{c}\text { Durée } \\
(\text { heures })\end{array}$ & $\begin{array}{c}\text { Epaisseur } \\
(\mu \mathrm{m})\end{array}$ \\
\hline A1 & 1100 & 1302 & 45 & 15 & 7 & 170 \\
\hline A2 & 1100 & 1316 & 45 & 15 & 7 & 240 \\
\hline A3 & 1100 & 658 & 45 & 15 & 7 & 100 \\
\hline A4 & 1100 & 658 & 18 & 6 & 7 & 100 \\
\hline A5 & 1100 & 1052 & 45 & 15 & 7 & 140 \\
\hline A6 & 1100 & 2632 & 45 & 15 & 7 & 285 \\
\hline
\end{tabular}

A $1350^{\circ} \mathrm{C}$ on obtient des revêtements de $\mathrm{Si}_{3} \mathrm{~N}_{4}-\alpha$ bien cristaliisé. Nous avons porté sur le Tableau II les conditions de dépôt et les résultats obtenus.

TABLEAU II

\begin{tabular}{|c|c|c|c|c|c|c|}
\hline $\begin{array}{c}\text { Echantilion } \\
\mathrm{N}^{*}\end{array}$ & $\begin{array}{c}\text { Temperature } \\
(\cdot \mathrm{C})\end{array}$ & $\begin{array}{c}\text { Pression } \\
(\mathrm{Pa})\end{array}$ & $\begin{array}{c}\mathrm{NH}_{3} \\
\mathrm{~N} . \mathrm{cm}^{3} / \mathrm{min}\end{array}$ & $\begin{array}{c}\text { T.M.S } \\
\mathrm{N} . \mathrm{cm}^{3} / \mathrm{min}\end{array}$ & $\begin{array}{c}\text { Duré } \\
(\text { heures })\end{array}$ & $\begin{array}{c}\text { Epaisseur } \\
(\mu \mathrm{m})\end{array}$ \\
\hline $\mathrm{C} 1$ & 1350 & 1052 & 18 & 6 & 2 & 351 \\
\hline $\mathrm{C} 2$ & 1350 & 658 & 24 & 8 & 6 & 263 \\
\hline $\mathrm{C} 3$ & 1350 & 658 & 24 & 8 & 6 & 267 \\
\hline $\mathrm{C4}$ & 1350 & 658 & 24 & 8 & 6 & 276 \\
\hline $\mathrm{C5}$ & 1350 & 658 & 24 & 8 & 6,5 & 310 \\
\hline $\mathrm{C} 6$ & 1350 & 658 & 24 & 8 & 3 & 155 \\
\hline
\end{tabular}

Les revêtements ainsi réalisés ont été caractérisés tout d'abord par diffraction de rayons $x$. On a pu confirmer que les revêtements obtenus à une température inférieure ou égale a $1100^{\circ} \mathrm{C}$ sont amorphes. Par contre ceux elaborés à $1350^{\circ} \mathrm{C}$ présentent des spectres de diffraction correspondant a la phase $\mathrm{Si}_{3} \mathrm{~N}_{4}-\alpha$.

L'examen au microscope échantilions A 1 et C 2 montre morphologie de surface est très électronique a balayage (MEB) des qu'on a deux séries de dépôts dont la differente suivant la temperature de 
préparation. Sur la figure 3a, on peut observer la surface d'un revêtement élaboré à $1100^{\circ} \mathrm{C}$. L'aspect "peau d'orange" est très caractéristique du $\mathrm{Si}_{3} \mathrm{~N}_{4}$ amorphe.

La figure $4 a$ est relative à la surface d'un dépôt obtenu à $1350^{\circ} \mathrm{C}$. cet échantillon présente une bonne cristallisation sous la forme de fins cristaux aux arêtes très vives.

L'analyse élémentaire, à la microsonde électronique, des revêtements amorphe $\mathrm{A} 1$ ou bien cristallisé $\mathrm{C2}$ fournit la composition equivalente à celle de $\mathrm{Si}_{3} \mathrm{~N}_{4}$ stoechiométrique. La seule impureté observée est l'oxygène dont la teneur reste très inférieure à $1 \%$.

\section{3-OBJECTIF ET CONDITIONS DES TRAITEMENTS THERMIOUES}

On recherche à mettre en évidence d'éventuelles modifications physico-chimiques, d'un dépôt de $\mathrm{Si}_{3} \mathrm{~N}_{4}$ sur son substrat, induites par des variations de température résultant de créneaux de flux radiatifs imposés à la surface sous atmosphere d'air ambiant. Les modifications attendues résultent, a priori, de phénomènes de décomposition, d'oxydation, de changements de texture et de phases cristallines. Toute dégradation mécanique notable décelable par les moyens de caractérisation mis en oeuvre sera donc, dans l'étude qui nous préoccupe, le signe que 1 'on se trouve en dehors du domaine d'intérêt pour les variables opératoires. La détermination de ce domaine a priori nécessiterait une connaissance thermique et thermo mécanique très complètes du système réel dépôt-substrat. Tout au plus, on peut avoir des ordres de grandeurs de certaines limites de températures qui seront imposées par la décomposition, l'oxydation, les dilatations respectives du dépôt et du substrat, la température d'élaboration qui constituera une limite inférieure du domaine étudié. Dans l'attente d'une modélisation thermo-mécanique du système entreprise par ailleurs, on procede à une exploration systématique des variables de contrôle des essais.

\section{4-ESSAIS THERMIQUES : CONDITIONS GENERALES}

Les traitements thermiques sont effectués à l'aide d'un four solaire de $6 \mathrm{~kW}$ à axe vertical dont les caractéristiques propres sont décrites en detail par ailleurs [17][18]. Le dispositif expérimental gue nous avons mis au point pour notre étude est schématisé figure 1. L'échantillon (A) placé dans son anneau de garde (B) reçoit un créneau de flux contrôlé par l'obturateur (C) programmé, à pales radiales. Un rétroviseur (D) permet les visées pour les mesures pyrométriques nécessaires pour la détermination de la température de surface du dépôt et de la loi temporelle exacte du créneau de flux. Le thermocouple (E) fixé sur la face arrière de l'échantillon permet de suivre l'évolution de la température de cellé-ci.

Le niveau de flux surfacique incident est sélectionné par positionnement de l'échantilion en élevation par rapport au foyer repéré du concentrateur. La durée choisie du créneau de flux est contrôlée par un micro processeur de commande du système actionneur de 1 'obturateur.

Le Elux réellement absorbé par l'échantillon est déterminé à partir de I'évaluation du flux réflechi par pyrométrie automatique (faible constante de temps) dans le spectre solaire : le même signal sert à déterminer la loi temporelle du créneau de flux.

La figure 2 présente un exemple des enregistrements obtenus pour le flux et les températures.

L'installation solaire ayant été étalonnée à l'origine [17] en flux incidents (par calorimetrie à l'aide d'un calorimetre a circulation d'eau et par radiométrie à l'aide de radiometres type Gardon) une simple vérification après nettoyage des facettes de l'heliostat est effectuée. On utilise un calorimètre a masse de cuivre soit en exploitant lélévation finale de température pour 1 'étalonnage de la fluence soit en exploitant la vitesse de variation de la température pour l'étalonnage du flux surfacique. La même methode est appliquée pour vérifier les flux absorbés déterminés par pyrométrie, en utilisant un échantillon de substrat (graphite) sans dépôt. On dispose ainsi de moyens d'essais, de contrôle et de mesures permettant d'effectuer les traitements thermiques des échantillons en utilisant deux variables opératoires : le flux surfacique de 100 a $450 \mathrm{w} / \mathrm{cm}^{2}$ 
et la durée du créneau, de quelques 1/10èmes de seconde à plusieurs minutes permettant d'atteindre sans difficulté des fluences de plus de $30 \mathrm{~kJ} / \mathrm{cm}^{2}$.
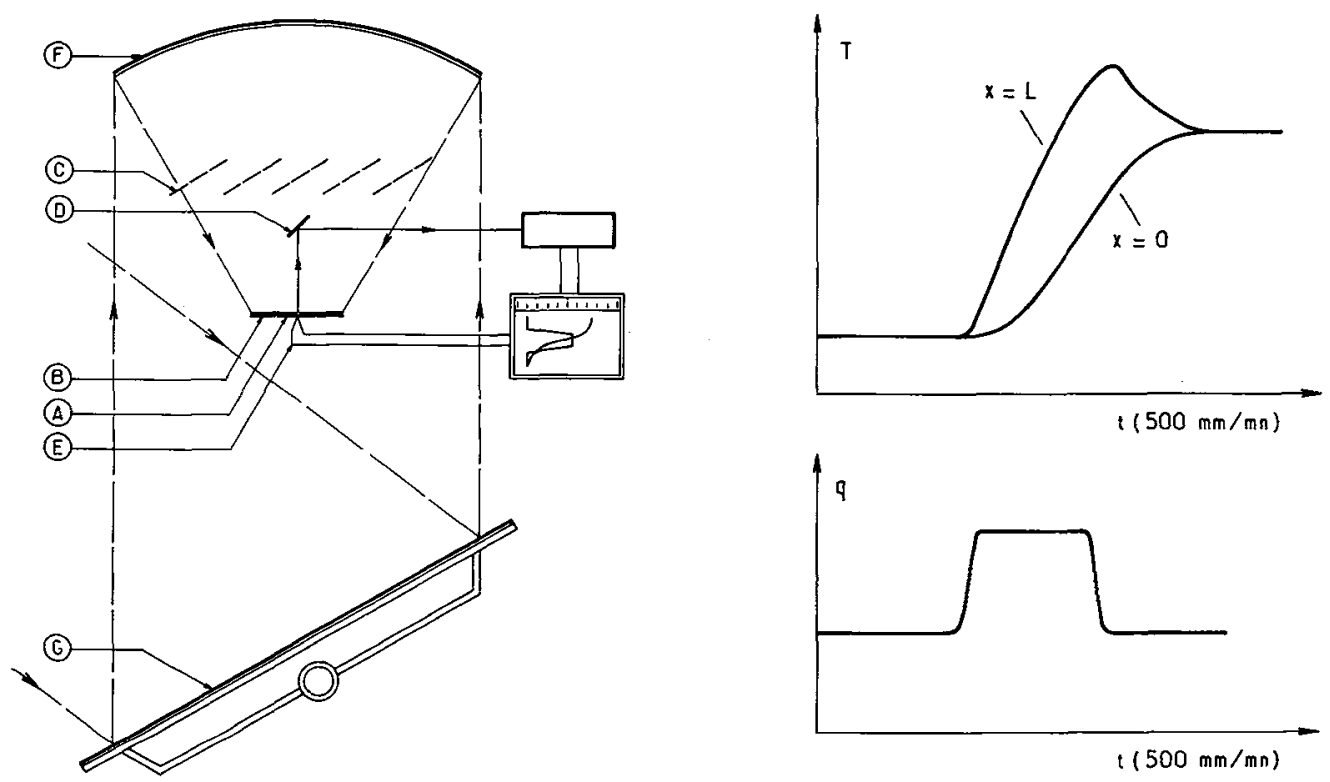

Fig.1 Dispositif expérimental de traitement thermique par concentration de rayonnement solaire. (A) échantilion, (B) anneau de garde, (C) obturateur, (D) rétroviseur, (E) thermocouple, (F) concentrateur, (G) héliostat.

Fig.2 Enregistrements types de flux surfacique $q$ et de température $T$ en fonction du temps $t$.

\section{5-TRAITEMENTS THERMIQUES DES DEPOTS}

5-1-Mode operatoire

A partir du lot d'échantillons disponibles on explore les domaines des variables operatoires du moyen d'essai : on utilise 4 niveaux de flux surfaciques à peu près uniformément repartis dans le domaine et des durées de créneaux permettant de couvrir un large domaine de températures maximales à la surface du dépôt.

Les moyens de caractérisations physico-chimiques des échantillons après essai ne permettent pas de réutiliser le même échantillon pour un nouvel essai thermique. Deux echantillons $A 1$ et $C 2$ supposés chacun représentatifs du lot de chaque série (amorphe et cristallisé) sont donc utilisés pour les caractérisations avant essai thermique.

5-2-Essais à basses températures.

Dans le cas du $\mathrm{Si}_{3} \mathrm{~N}_{4}-\alpha$ l'échantilion $\mathrm{Cl}$ est soumis à une série d'essais thermiques consécutifs aux differents niveaux de flux mais avec des fluences n'entrainant pas une température maximale sensiblement superieure a la température d'élaboration du dépôt : le tableau III rassemble les données et observations concernant ces essais.

TABLEAU III

\begin{tabular}{|c|c|c|c|c|c|c|}
\hline Essai N $\mathrm{N}^{*}$ (Echantillon Cl) & 1 & 2 & 3 & 4 & 5 & 6 \\
\hline Flux incident sur 1'échantillon $\left(\mathrm{W} / \mathrm{cm}^{2}\right)$ & 395 & 410 & 304 & 203 & 100 & 98 \\
\hline Durée de l'essai (s) & 10 & 15 & 20 & 25 & 35 & 60 \\
\hline Température de surface $\left(^{\circ} \mathrm{C}\right)$ & 1080 & 1300 & 1400 & 1210 & 865 & 1050 \\
\hline Température à $\mathrm{I}^{\prime}$ arrière $\left({ }^{\circ} \mathrm{C}\right)$ & 850 & 1150 & 1205 & 1065 & 735 & 970 \\
\hline
\end{tabular}


5-3-Essais a hautes températures

Chaque échantilion restant disponible ( $S i_{3} N_{4}-\alpha$ ou amorphe) est alors soumis à un seul traitement thermique conduisant a une temperature maximale de surface supérieure a la temperature d'élaboration du dépôt concerné. Les données et observations concernant ces essais sont rassemblees tableaux IV et $V$.

TABLEAU IV

\begin{tabular}{|c|c|c|c|c|c|c|}
\hline Echantillon $\mathrm{N}^{\circ}$ & A1 & A2 & A3 & A4 & A5 & $\overline{A 6}$ \\
\hline Flux incident $\left(\omega / \mathrm{cm}^{2}\right)$ & 99 & 113 & 214 & 370 & 432 & 421 \\
\hline Durée de l'essai (s) & 100 & 250 & 50 & 15 & 20 & 15 \\
\hline Temp $\max \left({ }^{\circ} \mathrm{C}\right)$ & 1200 & 1500 & 1700 & 1350 & 1930 & 1800 \\
\hline $\begin{array}{c}\text { Diffraction } \\
\text { Rayons } \mathrm{X}\end{array}$ & inchangé & inchangé & $\mathrm{Si}_{3} \mathrm{~N}_{4}-\alpha$ & inchangé & $\begin{array}{l}\mathrm{Si}_{3} \mathrm{~N}_{4}-\alpha \\
\mathrm{Si}_{3} \mathrm{~N}_{4}-\beta\end{array}$ & $\begin{array}{l}\operatorname{si}_{3} N_{4}-\alpha \\
\operatorname{si}_{3} N_{4}-\beta\end{array}$ \\
\hline Observations & $\begin{array}{c}\text { couleur } \\
\text { peu } \\
\text { changée }\end{array}$ & $\begin{array}{c}\text { violet } \\
\text { fissures }\end{array}$ & $\begin{array}{c}\text { violet } \\
\text { fissures }\end{array}$ & $\begin{array}{l}\text { couleur } \\
\text { changée } \\
\text { fissures }\end{array}$ & $\begin{array}{c}\text { violet } \\
\text { fissures }\end{array}$ & $\begin{array}{c}\text { violet } \\
\text { fissures }\end{array}$ \\
\hline
\end{tabular}

TABLEAU $v$

\begin{tabular}{|c|c|c|c|c|c|}
\hline Echantilion $\mathrm{N}^{\circ}$ & C3 & $\mathrm{C} 4$ & $\mathrm{C4}$ & $\mathrm{C5}$ & $\mathrm{c6}$ \\
\hline Flux incident $\left(\mathrm{w} / \mathrm{cm}^{2}\right)$ & 425 & 103 & 99 & 208 & 425 \\
\hline Durée de l'essai (s) & 20 & 106 & 252,5 & 50 & 16,5 \\
\hline Temp.max. $\left({ }^{\circ} \mathrm{C}\right)$ & 2030 & 1410 & 1490 & 1740 & 1910 \\
\hline Observations & $\begin{array}{c}\text { se fissure } \\
\text { se décompose }\end{array}$ & $\begin{array}{c}\text { pas de } \\
\text { changement }\end{array}$ & $\begin{array}{c}\text { pas de } \\
\text { changement }\end{array}$ & $\begin{array}{c}\text { violet } \\
1 \text { fissure }\end{array}$ & $\begin{array}{c}\text { un peu } \\
\text { décomposé }\end{array}$ \\
\hline Diffrac. Ray. $\mathrm{X}$ & inchangé & inchangé & inchangé & inchangé & inchange \\
\hline
\end{tabular}

5-4-Caractérisation des échantillons après essais

Après avoir subi le traitement thermique, tous les echantilions sont examines au microscope electronique a balayage (MEB). IIs subissent egalement les caracterisations classiques : diffraction de rayons $X$ et analyse par microsonde électronique et spectroscopie AUGER.

\section{6-PRESENTATION ET DISCUSSION DES RESULTATS}

\section{6-1-Observations au MEB}

Nous avons vu au paragraphe 2 que suivant les conditions de préparation nous obtenons deux variétés différentes qu'on peut observer sur les figures $3 a$ et $4 a$.

Ces deux séries d'échantillons ont subi un traitement thermique et nous avons observé au MEB les modifications de la surface. Sur la figure $3 b$ on peut constater que 1 'échantilion $A 3$ qui a été soumis pendant 50 s an flux de $214 \mathrm{~W} / \mathrm{cm}^{2}$ et a atteint une temperature de surface maximum $\mathrm{T}_{\mathrm{max}}=1700^{\circ} \mathrm{C}$ présente de nombreuses fissures. La figure 3c montre le détail de 1 'intersection de deux fissures, en partie colmatee par une mince couche de silice (d'après analyse).

Avec des conditions de flux et de température supérieures (2Os à 432 $w / \mathrm{cm}^{2}$ et $T_{\text {max }}=1930^{\circ} \mathrm{C}$ ) nous pouvons observer Fig. $3 \mathrm{~d}$ que la surface et les micro fissures sont en partie recouvertes d'une mince couche de silice.

Les observations sur les échantillons cristaliisés sont présentées sur la figure 4.

Ia figure $4 b$ montre I'échantilion $c 6$ traité $16,5 s$ sous un flux de $425 \mathrm{~W} / \mathrm{cm}^{2}$ qui a conduit à une $\mathrm{T}_{\mathrm{max}}=1910^{\circ} \mathrm{C}$. On constate que les arêtes des cristaux sont considerablement arrondies. Il semblerait qu'il y ait eu transport de matière du sommet au pied des cristaux.

Sur la figure $4 \mathrm{c}$, relative à l'échantilion $\mathrm{c} 3$, on constate une importante fissuration après une exposition de 20 s sous un $f l u x$ de $425 \mathrm{w} / \mathrm{cm}^{2}$ 
a $\quad T_{\max }=2030^{\circ} \mathrm{C}$. A haute température $\mathrm{Si} \mathrm{N}_{4}$ réagit avec $I^{\prime}$ air pour donner une couche de silice. La figure $3 d$ illustre ce phénomene.

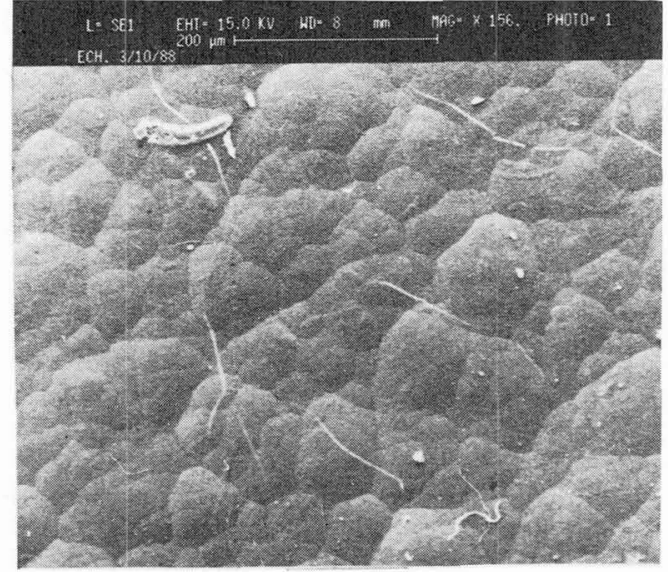

(a)

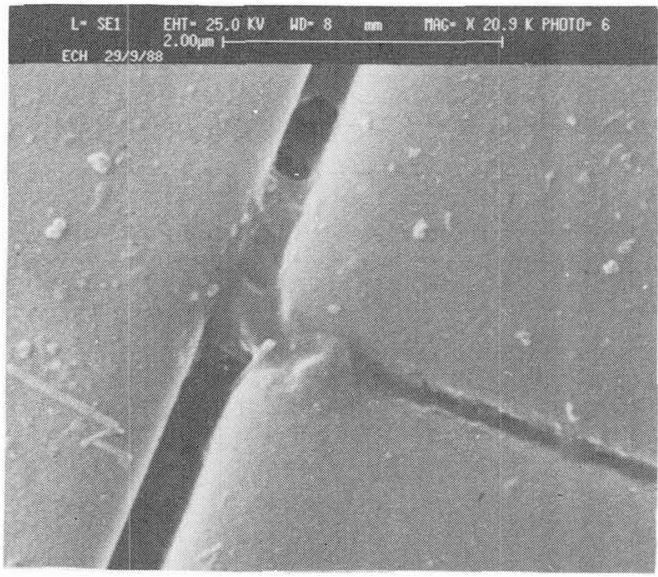

(c)

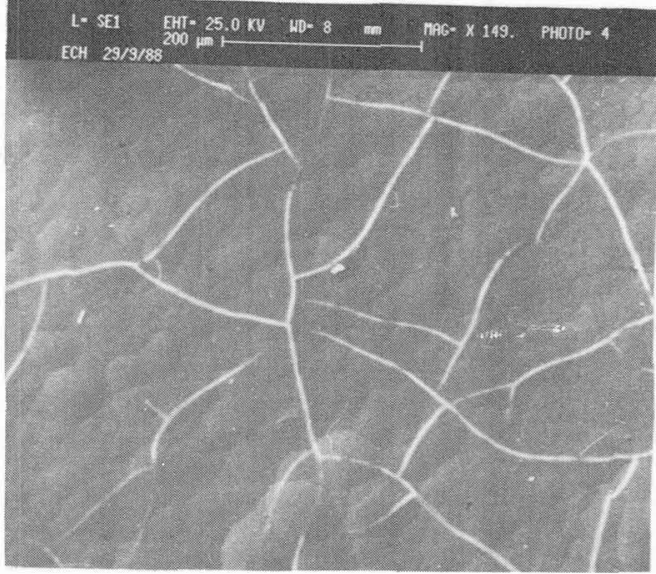

(b)

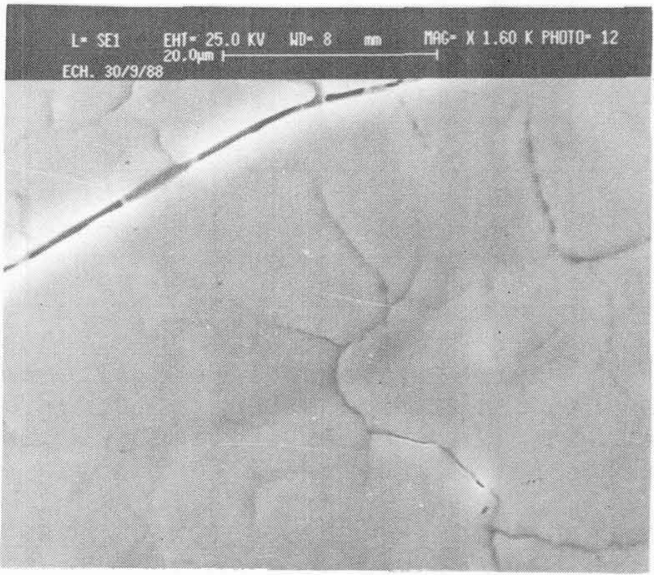

(d)

Fig. 3 Observations au MEB : (a) surface de l'échantillon A1; (b) surface de l'échantillon A3 (50s, flux $214 \mathrm{~W} / \mathrm{cm}^{2}, T_{\text {max }}=1700^{\circ} \mathrm{C}$ ); (c) détail d'une Eissure de l'échantilion A3; (d) surface de l'échantillon A5 (20s, flux: $432 \mathrm{~W} / \mathrm{cm}^{2}, T_{\max }=1930^{\circ} \mathrm{C}$ ).

6-2-Domaines de comportement thermo-mécanique

On a reporté figure 5 les résultats des traitements thermiques des deux types de couches présentés tableaux III à V. Les points expérimentaux pour $\mathrm{Si}_{3} \mathrm{~N}_{4}-\alpha$ sont placés dans un diagramme fluence/flux surfacique. La température maximale atteinte en surface lors de l'essai figure entre parenthèses pour chaque point. L'apparition de fissures est noté par un signe $(x)$ accompagnant le symbole du point. Des limites approximatives ont été tracées à partir des points expérimentaux ; ces limites signifient qu'au dela des valeurs des variables operatoires correspondantes il y a apparition de fissures qui témoignent d'une dégradation notable des propriétes mécaniques des revêtements.

Le matériau amorphe sur son substrat peut résister a des flux surfaciques de $400 \mathrm{~W} / \mathrm{cm}^{2}$ à condition que la fluence ne dépasse pas environ 5 
$\mathrm{kJ} / \mathrm{cm}^{2}$ : la température de surface doit rester inférieure à $1350^{\circ} \mathrm{C}$. $\mathrm{Si}_{3} \mathrm{~N}_{4}-\alpha$ est sensiblement plus résistant : il a un bon comportement sous $200 \mathrm{~W} / \mathrm{cm}^{2}$ en deça de $10 \mathrm{~kJ} / \mathrm{cm}^{2}$ soit une température limite inférieure à $1740^{\circ} \mathrm{C}$; il peut également résister à des flux de 1 'ordre de $400 \mathrm{w} / \mathrm{cm}^{2} \mathrm{mais}$ avec des fluences ne dépassant pas $7 \mathrm{~kJ} / \mathrm{cm}^{2}$ soit $\mathrm{T}_{\mathrm{m} \text { x }}=1910^{\circ} \mathrm{C}$. De plus,

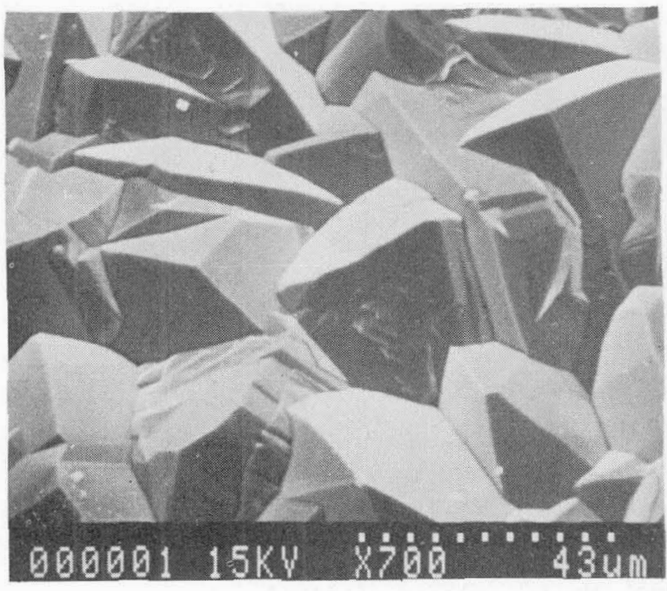

(a)

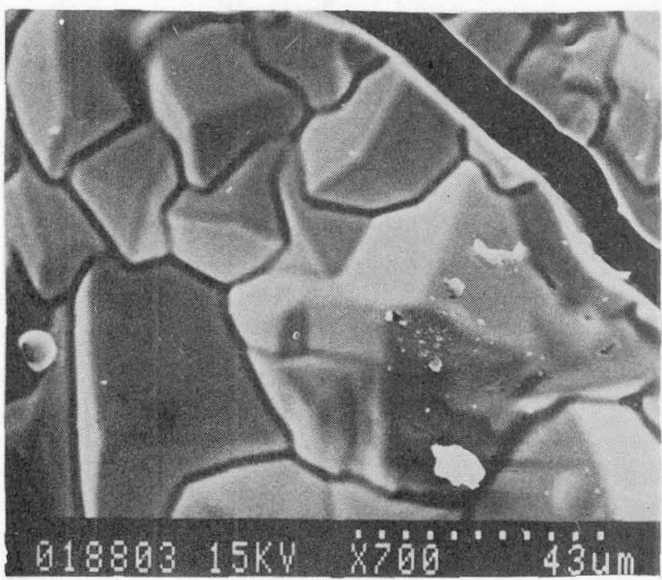

(c)

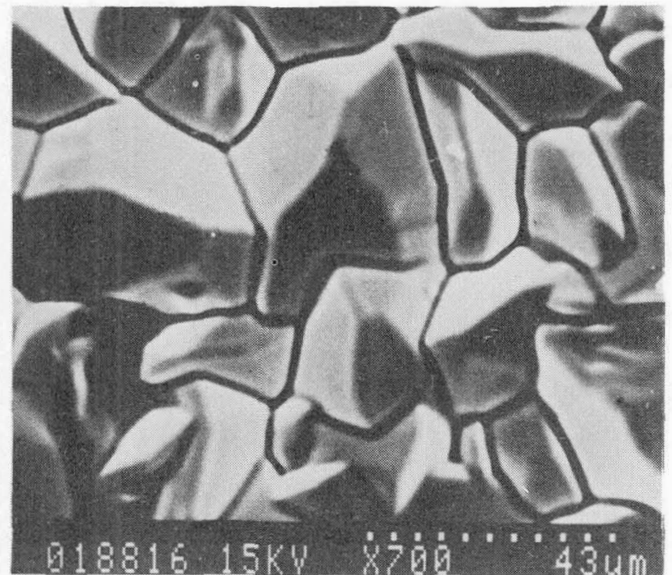

(b)

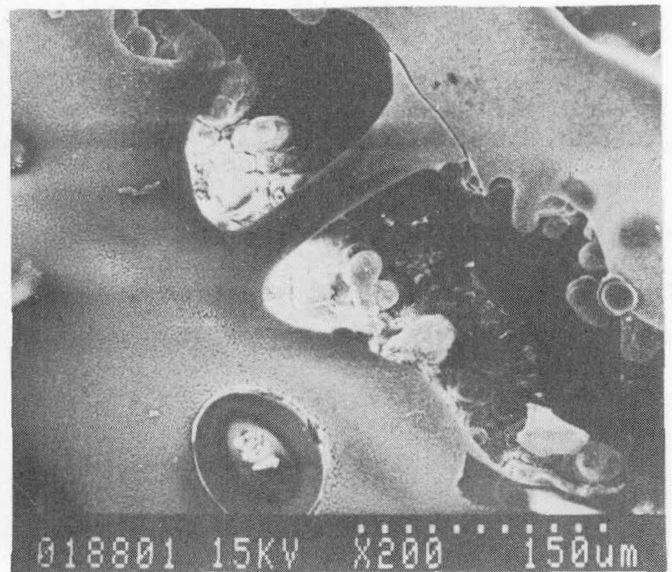

(d)

Fig.4 Observations au MEB : (a) surface de l'échantillon C2; (b) surface de l'echantilion $\mathrm{C} 6 \quad\left(16,5 \mathrm{~s} ;\right.$ flux: $\left.425 \mathrm{~W} / \mathrm{cm}^{2} ; \quad \mathrm{T}_{\mathrm{max}}=1910^{\circ} \mathrm{C}\right) ;$ (c) échantilion $\mathrm{C} 3$ avec fissure $\left(20 \mathrm{~s}\right.$, flux: $425 \mathrm{~W} / \mathrm{cm}^{2}$ $\left.T_{\max }=2030^{\circ} \mathrm{C}\right)$; (d) région fortement dégradée de $I^{\prime}$ échantilion $\mathrm{C} 3$.

I'échantillon $\mathrm{Cl}$ de $\mathrm{Si}_{3} \mathrm{~N}_{4}-\alpha$ soumis à 6 traitements successifs (tableau III) couvrant le domaine de flux surfaciques disponibles ne manifeste aucune dégradation notable tant que la fluence reste voisine de $5 \mathrm{~kJ} / \mathrm{cm}^{2}$ soit $\mathrm{T}_{\mathrm{max}}=$ $1400^{\circ} \mathrm{C}$.

Il convient de remarquer que le flux surfacique et la fluence sont bien les variables operatoires des essais thermiques mis en oeuvre : nous avons utilisé les mêmes variables pour caractériser le comportement des couches sur leur substrat.Ceci peut être interessant pour certaines applications dans des cas d'environnements radiatifs sévères ; il faut 
cependant garder à l'esprit que les résultats obtenus sont relatifs à des couches sur leur substrat constituant un système thermique défini. Une représentation des résultats, avec une portée moins limitée, pourrait être obtenue en utilisant les variables $T_{\max }$ et ( $\partial \mathrm{T} / \partial \mathrm{t}$ ) max a la surface correspondant respectivement à la fluence et au flux surfacique ; mais, dans nos essais, les mesures de températures de surface peuvent être entachées d'erreurs telles qu'il nous est apparu préférable d'utiliser les variables fluence et flux surfacique determinées avec une bonne précision (incertitude inférieure à 5\%).

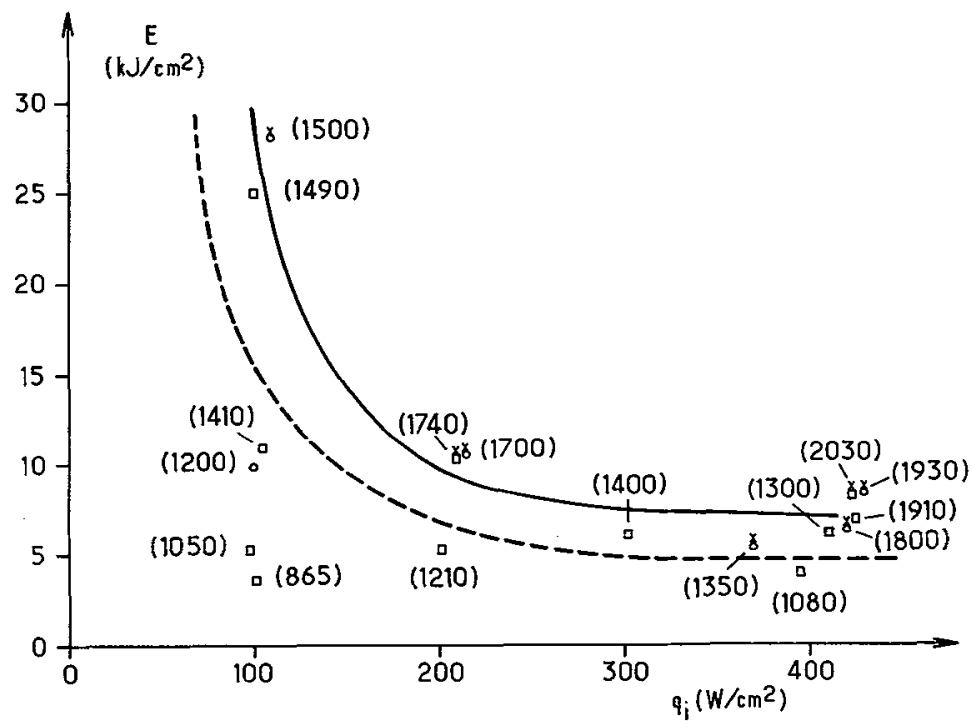

Fig.5 Résultats du comportement des dépôts de $\mathrm{Si}_{3} \mathrm{~N}_{4}$ dans un diagramme fluence/flux surfacique:---O dépôt $\mathrm{Si}_{3} \mathrm{~N}_{4}$ amorphe; -0 dépôt $\mathrm{Si}_{3} \mathrm{~N}_{4}-\alpha$; $x$ dépôt présentant des fissures après traitement; ( ) température maximum atteinte $\left({ }^{\circ} \mathrm{C}\right)$.

6-3-Caractérisation par diffraction de rayons $x$

Tous les échantilions subissent un examen par diffraction de rayons $\mathrm{X}$ avant et après traitement thermique.

Cas du materiau amorphe

On constate une recristallisation du matériau sous forme de $\mathrm{Si}_{3} \mathrm{~N}_{4}-\alpha$ pour une durée d'exposition de $50 \mathrm{~s}$, un flux de $214 \mathrm{~W} / \mathrm{cm}^{2}$ et $T_{\text {max }}=1700^{\circ} \mathrm{C}$. Lorsque la temperature atteint ou dépasse $T_{\max }=1800^{\circ} \mathrm{C}$, il $y$ a apparition simultanée des deux formes cristallines $\mathrm{Si}_{3} \mathrm{~N}_{4}{ }^{-\alpha}$ et $\beta$. La couche de silice formée en surface ne donne pas de raies de diffraction et semble donc amorphe.

Cas du matériau cristallisé

Dans ce cas on ne constate pas de modification sensible de la structure cristalline du matériau. En particulier, on n'a pas observé de formation de $S i_{3} N_{4}-\beta$ comme dans le cas de 1 'amorphe. Il faut cependant noter qu'une étude fine des diagrammes de diffraction n'est pas aisé, car les diffractogrammes sont réalisés sur les surfaces vapodéposées ou traitées thermiquement et non pas sur une poudre. Donc cette étude ne peut être que qualitative. La couche de silice ne donne pas ici non plus de raies de diffraction et semble donc amorphe. 
6-4-Analyses à la microsonde électronique et par spectroscopie

Auger.

Des analyses à la microsonde électronique ont été effectués sur les échantillons ayant subi le traitement thermique. L'etude est réalisée sur les coupes perpendiculaires à la surface traitée et convenablement polies. Il faut signaler que nous $n$ avons pas pu analyser les echantillons amorphes, car après traitement thermique la fissuration est trop importante. On présente ici la variation des concentrations en silicium et azote lorsqu'on va de la surface vers l'intérieur dans le cas de l'échantillon C5. La concentration en silicium reste constante dans tout 1'échantillon. Par contre, la concentration en azote diminue nettement pres de la surface sur une profondeur d'environ $15 \mu \mathrm{m}$. Ce qui indique une décomposition du $\mathrm{Si}_{3} \mathrm{~N}_{4}-\alpha$ en surface. Sur la figure 6 nous donnons la variation de l'intensité du signal en fonction de la profondeur (d $\mathrm{dm}$ ) dans le cas de ces deux éléments.

L'oxygène n'est présent que sur la surface et sur les bords des fissures sous forme de silice amorphe et ne semble donc pas diffuser en profondeur lors du traitement thermique.

L'analyse de la surface des échantillons par spectrométrie Auger a permis d'identifier un film de $\mathrm{SiO}_{2}$ recouvrant le $\mathrm{Si}_{3} \mathrm{~N}_{4}$ : raies du silicium a $76 \mathrm{ev}$ et de l'oxygène à $487 \mathrm{ev}$. Le mécanisme d'oxydation de PDSN a déà eté largement abordé par plusieurs auteurs [12-13-14].

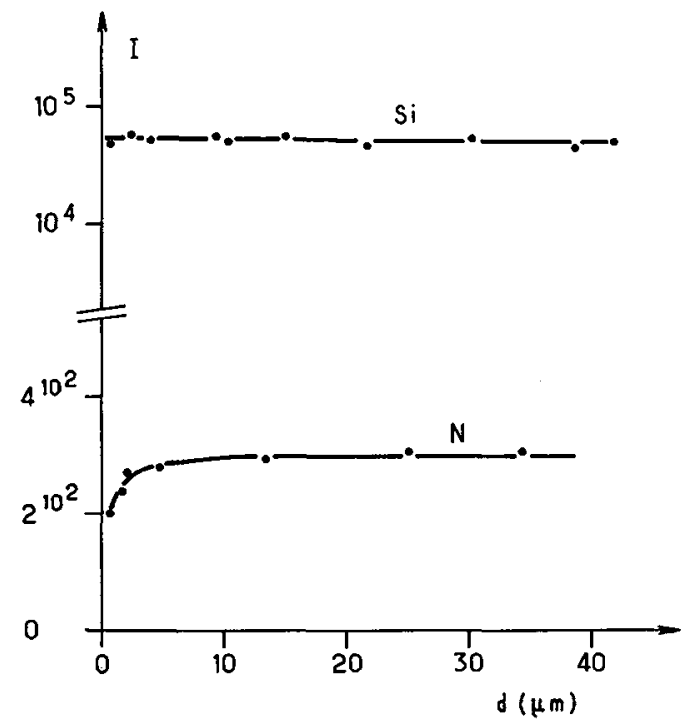

Fig.6-Analyse à la microsonde électronique d'une coupe perpendiculaire à la surface traitée : (I) nombre de coups par seconde pour le silicium et l'azote en fonction de la distance (d $\mu \mathrm{m}$ ) a partir de la surface traitée thermiquement.

\section{7-CONCLUSION}

Les deux varietes de PDSN soumises aux traitements thermiques mis en oeuvre manifestent des comportements physico-chimiques differents.

On constate, dans les conditions des essais, que la variété $\mathrm{Si}_{3} \mathrm{Ni}_{4}-\alpha$ ne subit pas de transformation de phase jusqu'à $2030^{\circ} \mathrm{C}$ au moins ; la variété $\mathrm{Si}_{3} \mathrm{~N}_{4}$ amorphe, quant à elle, se transforme partiellement ensi $\mathrm{N}_{4}-\alpha$ des $1700^{\circ} \mathrm{C}$ puis en un mélange de $\mathrm{Si}_{3} \mathrm{~N}_{4}-\alpha$ et $\beta$ dès $1800^{\circ} \mathrm{C}$.

Les courches de $\mathrm{Si}_{3} \mathrm{~N}_{4}$ amorphe se fissurent abondamment des $1350^{\circ} \mathrm{C}$ quel que soit le niveau de flux surfacique; la fissuration des couches de $\mathrm{Si}_{3} \mathrm{~N}_{4}-\alpha \mathrm{n}^{\prime}$ intervient qu'à partir de $1740^{\circ} \mathrm{C}$; de plus une couche de $S i_{3} \mathrm{~N}_{4}-\alpha$ 
soumise à des créneaux successifs jusqu'au niveau maximum disponible de 450 $W / \mathrm{cm}^{2}$ ne montre pas de dégradation notable tant que la température maximum atteinte en surface ne dépasse pas $1400^{\circ} \mathrm{C}$ : il est à noter que cette temperature est voisine de la température d'élaboration de la variété si $\mathrm{N}_{4}$ $\alpha$. Il apparait donc que cette température est une caractéristique importante du comportement au choc thermique des deux variétés de PDSN étudiées.

Les essais a flux surfaciques et fluences variables conduits sur ces deux matériaux ont permis de mettre en évidence une limite approximative pour chacun d'un domaine de variables opératoires au delà duquel la dégradation thermomécanique par fissuration devient notable. Dans le domaine en deca de cette limite seules des transformations physico-chimiques du type décomposition oxydation apparaissent : perte d'azote en surface et formation d'un film de $\mathrm{SiO}_{2}$ amorphe à haute temperature. Ce film de sio amorphe peut expliquer en partie l'absence de diffusion de l'oxygène en profondeur dans la couche qui peut aussi résulter de la nature même des couches (PDSN dense) et de la forme impulsionnelle des traitements thermiques. on note par ailleurs que dans le cas du matériau $S i{ } N_{4}$ amorphe les fissures très fines et abondantes apparaissant à partir de $1350^{\circ} \mathrm{C}$ sont pratiquement colmatés par lefilm de $\mathrm{SiO}_{2}$ à haute température ce qui n'est pas le cas pour les fissures du $\mathrm{Si}_{3} \mathrm{~N}_{4}-\alpha$.

Un prolongement intéressant de ce travail devrait porter sur l'exploration des flux surfaciques supérieurs à la limite disponible dans cette etude et sur l'évaluation du comportement de ces couches soumises à des cycles de traitements thermiques pour déterminer si les transformations induites font apparaftre une amélioration dans la resistance aux chocs thermiques et à l'oxydation de ces couches.



\section{BIBLIOGRAPHIE}

[1] Hasselman D.P.H., J.Am.Ceram.Soc. 62, (3-4), (1979) 125

[2] Faber K.T.,Huang M.D.,Evans A.G., J.Am.Cer.Soc. 64, (5), (1981) 296

[3] Majdic A., Science of Ceramics 12, (1983) 39

[4] Lamon J., Rev. Int. Hautes Temper Refract., Fr., 22, (1985) 115

[5] Hasselman D.P.H., J.Am.Cer.Soc. 46 (5), (1963) 229

[6] Walton J.D, Royère C., Science of Ceramics 7, (1973) 219

[7] Carrie J.P, Le Doussal H, Chastant M, Halm L, Jon M, Royere C, Tuhault J.L., Bull.Soc.Fr.Ceram. 24, (1979) 29

[8] Millard J.M, Miyake R.N, Dirling R.B., Rolfo A., Royère C., Int.Symp. on. "Environmental and thermal systems for space vehicles"TOULOUSE (4-7) oct.1983), Proc.531-560 (ESA SP 200, Dec 1983)

[9] Royère C., Cahiers AFEDES, 3, (Avril 1985) 171

[10] Rice R.W, Freiman S.W, Mecholsky J.J, Ruh R, Harada Y., Ceramics for High Performance Application II - Proc. Fifth Army Materials Technology Conference, (Newport, Rh.Isl., 21-25 March 1977), (Ed.J.J.BURKE, E.N.LENOE, R.W.KATZ), Brook Hill Publ.Co., 1978, $669 / 687$.

[11] Chermant J.L., Moussa R., Osterstock F.,Rev.Int.Hautes Temper. Réfract, $\underline{18},(19.81) 5$

[12] Singhal S.C., J. of Mater. Science. 11, (1976) 500

[13] Hirai T., Nithara K., Goto T., J.Am.Ceram.Soc.63, (7/8), (1980) 419

[14] Lartigue J.F.- Thèse Docteur Ingénieur- Univ. de Grenoble, (1985)

[15] Combescure C., Armas B., Coll.Intern. sur les matériaux durs pour frottement dans les réacteurs nucléaires, Avignon, Ed.S.F.E.N., (1980) 65

[16] Lartigue J.F., Ducarroir M., Armas B., Proc. of the 9th Int.Conf. on CVD., Ed. The Electrochem. Soc. 84 (6), (1984) 561

[17] Olalde G.. Thèse de Docteur ès Sciences Physiques, Université de Perpignan, (1981)

[18] Arnaud G., Flamant G., Olalde G., Robert J.F.,Entropie, 97 (1981) 139 